\title{
Adaptive Interpupillary Distance Adjustment for Stereoscopic 3D Visualization
}

\author{
Hyungon Kim \\ HIT Lab NZ \\ University of Canterbury \\ Christchurch, New Zealand \\ hyungon.kim \\ @pg.canterbury.ac.nz
}

\author{
Gun Lee \\ HIT Lab NZ \\ University of Canterbury \\ Christchurch, New Zealand \\ gun.lee \\ @canterbury.ac.nz
}

\author{
Mark Billinghurst \\ HIT Lab NZ \\ University of Canterbury \\ Christchurch, New Zealand \\ mark.billinghurst \\ @canterbury.ac.nz
}

\begin{abstract}
Stereoscopic visualization creates illusions of depth through disparity between the images shown to left and right eyes of the viewer. While the stereoscopic visualization is widely adopted in immersive visualization systems to improve user experience, it can also cause visual discomfort if the stereoscopic viewing parameters are not adjusted appropriately. These parameters are usually manually adjusted based on human factors and empirical knowledge of the developer or even the user. However, scenes with dynamic change in scale and configuration can lead into continuous adjustment of these parameters while viewing. In this paper, we propose a method to adjust the interpupillary distance adaptively and automatically according to the configuration of the $3 \mathrm{D}$ scene, so that the visualized scene can maintain sufficient stereo effect while reducing visual discomfort.
\end{abstract}

\section{Categories and Subject Descriptors}

I.3.7 [Computer Graphics]: Three-Dimensional Graphics and Realism - Virtual reality.

\section{General Terms}

Measurement, Design, Experimentation, and Human Factors.

\section{Keywords}

3D stereoscopic images, interpupillary distance (IPD), and visual discomfort/comfort.

\section{INTRODUCTION}

Many researches in the field of virtual reality developed a variety of display hardware and visualization methods to provide visually immersive experience. One of these technique is 3D stereoscopic visualization which is recently and actively being adopted in Televisions, movie theaters, entertainment industry and human computer interface research [1][2][3]. Compared to traditional two dimensional displays, 3D stereoscopic visualization can create an enhanced user experience [4][5] since it is able to provide the perception of depth of the visualized scene [6].

However, 3D stereoscopic visualization can produce visual discomfort as well. In previous work on 3D stereoscopic safety, parallax distribution, depth inconsistency, vergence accommodation conflict, and binocular mismatches were identified as the factors that can trigger visual discomfort and fatigue [7], and various approaches have been investigated to reduce the visual discomfort and fatigue with immersive 3D stereoscopic visualization.

One of the main factors that cause visual discomfort in stereoscopic visualization is extreme or incorrect disparity between the views shown to the left and right eyes of the user [9]. The image disparity results from visualizing the 3D scene from two different perspectives corresponding to each eye of the user. The two images generated for each eye depends on various factors, such as the interpupillary distance (IPD), convergence, the distance between a viewer and a scene, the scale of a 3D scene, the size of the display screen, intrinsic camera parameters (such as focal length, coordinates of the images, and radial distortion) and extrinsic camera parameters (camera position and the direction of its optical axis) [15]. If these factors are not properly adjusted according to the viewing environment and human factors, the resulting stereoscopic image can have extreme or incorrect disparity, causing visual discomfort and unrealistic scenes. Figure 1 shows an example of stereoscopic visualization with extreme disparity that results in very different images shown to each eye.

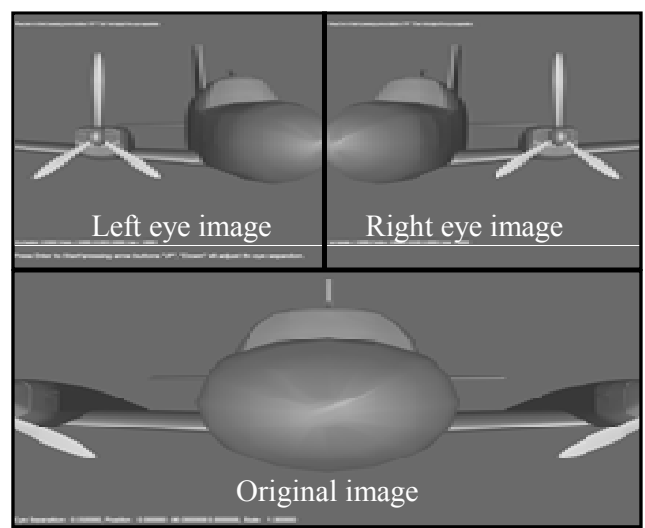

Figure 1. Stereoscopic visualization with extreme disparity.

Among the factors, IPD (a.k.a. interocular distance or baseline) is the most widely used parameter to adjust the amount of stereoscopic effect [8][12][13]. IPD represents the separation 
between the two eyes, and it is also used for describing how far the two cameras are displaced to produce stereoscopic images. When the IPD is set to zero, the resulting image shows no stereoscopic effect as the images for each eye become identical. Increasing the IPD gives more depth illusion to the user as the disparity between the two images grows. And if it is raised above a certain level, the user starts to feel eyestrain and eventually it becomes hard to see the image correctly (e.g. resulting in double vision). Therefore, the IPD has to be adjusted carefully in order to provide sufficient stereo effect while also maintaining user comfort.

When visualizing 3D scenes that dynamically change in scale or distance, the IPD needs to be adjusted according to the scene configuration. However, in many cases, it is manually set to a fixed value throughout the content. In this paper, we propose a method to adaptively and automatically adjust the IPD according to the configuration of the $3 \mathrm{D}$ scene, so that the visualization can maintain sufficient stereo effect while reducing visual discomfort.

\section{RELATED WORK}

Previous research has investigated how to reduce visual discomfort in viewing stereoscopic images in various configurations. These included investigating parallax distribution patterns in the image space [10], reducing the baseline for stereo cameras on mobile [8], and generating in-between virtual views to reduce parallax [12][13]. In all these cases, the appropriate IPD is manually set by those who are preparing and producing the content, or by the viewers themselves.

For example, NVIDIA 3D Vision stereo glasses provide a knob on the IR emitter with which the user can manually adjust the IPD to reduce visual fatigue. The system also provides a set of profiles for 3D software applications, so that the appropriate IPD can be applied for different software.

There has also been previous work on adaptively adjusting stereo visualization parameters at production time according to the screen sizes [9][11] or the intended region of interest in depth space [14]. However, to our best knowledge, there has been no previous work adjusting IPD at runtime adaptively according to the scene configuration (especially, relative distance between the scene and the user's viewpoint).

\section{ADAPTIVE INTERPUPILLAR DISTANCE ADJUSTMENT}

Proper IPD for visualizing the stereoscopic images of a 3D scene is decided by many different factors including, physical IPD of the user, physical size of the screen, and size or distance of the 3D scene relative to the user's viewpoint. Among these factors, the size and distance of the 3D scene relative to user's viewpoint are the factors that can change dynamically depending on the content.

For instance, when a virtual earth is shown from a distance, in order to provide enough depth perception, the IPD should be in scale of the radius of the earth. On the other hand, when the same virtual earth is shown from the ground level viewpoint (e.g. showing street level view), the IPD should be scaled down accordingly, otherwise the image disparity will become too extreme causing visual discomfort.
Based on this heuristic observation, we propose to automatically adjust the IPD according to the distance between the scene and the user's viewpoint.

To demonstrate the feasibility of the proposed method, we implemented a prototype stereoscopic 3D visualization system. For displaying 3D stereoscopic images, we used '3D Vision Ready' active stereoscopic shutter glasses with Samsung 22 inch $120 \mathrm{~Hz}$ 3D monitor on an Intel Core i7 $3.4 \mathrm{GHz}$ based computer equipped with Nvidia Geforce GTX670 graphics card. We used the Open Scene Graph library (http://www.openscenegraph.org) for real-time 3D scene rendering with its feature supporting quadbuffered stereo.

The system allowed the IPD to be adjusted both manually and automatically in order to investigate the proposed method through a user experiment.

\section{USER EXPERIMENTS}

We conducted two user experiments to show the feasibility of the proposed method. First, in order to determine the appropriate IPD corresponding to the distance between the user and a 3D scene, we conducted a user experiment which asked users to choose proper IPD for varying distance between the scene and the user's viewpoint. Second, we compared the level of visual discomfort and subjective depth perception between three different visualization configurations: (1) monoscopic visualization, (2) stereoscopic visualization with fixed IPD, and (3) stereoscopic visualization with adaptive IPD.

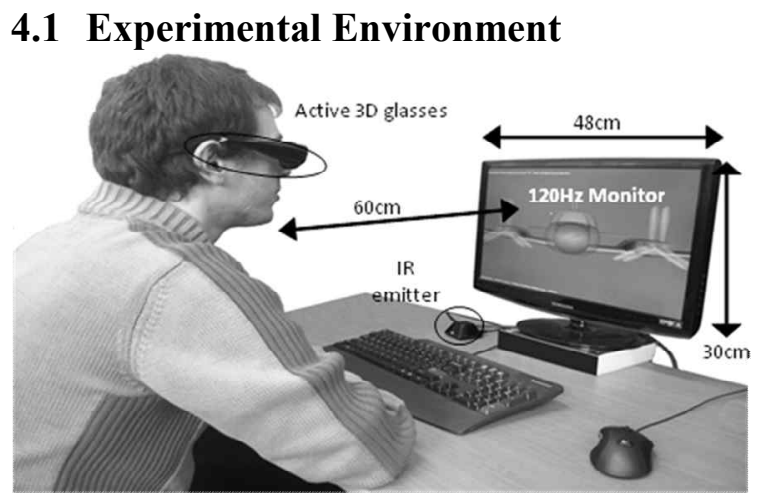

Figure 2. Experimental Environment

Figure 2 shows the experimental environment. We used the prototype stereoscopic visualization system described in section 4 . The participants sat approximately $60 \mathrm{~cm}$ away from the monitor wearing the stereo glasses.

Figure 3 illustrates the 3D environment used in both of the experiments. Dotted lines represent the view frustum used for the left and right eyes in stereoscopic visualization, and the solid lines show the view frustum used for the monoscopic visualization. For the 3D scene, we used an airplane model with fairly complex geometries. The airplane model was initially located at 100 units (1 unit $=$ approximately $0.5 \mathrm{~cm}$ ) away from the projection plane, and was moved towards the user or further away during the experiment. 


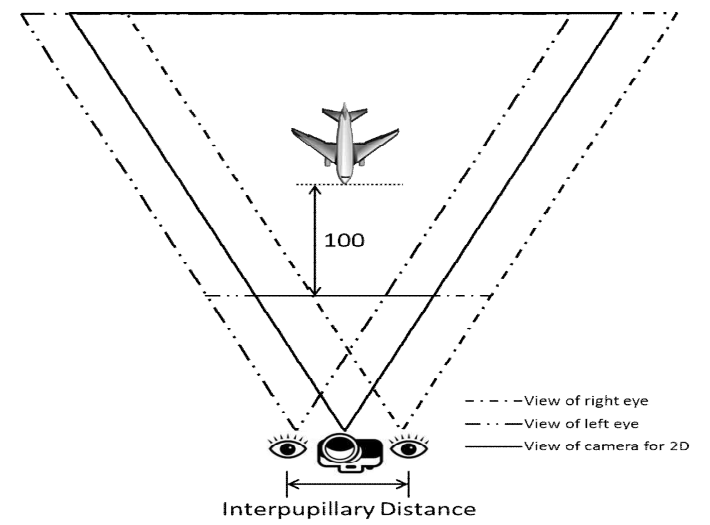

Figure 3. 3D scene configuration for the experiments

\subsection{Experiment I}

\subsubsection{Experimental Procedure}

In the first experiment, to measure appropriate IPD with respect to the location of the 3D object, we measured IPDs that participants perceived it provided sufficient stereoscopic effect yet comfortable to their eyes.

At the beginning of the experiment, the 3D model was shown at its initial location, and the IPD was set to 6.5 centimeters $(\mathrm{cm})$. The participants were asked to adjust the IPD by pressing the arrow keys on the keyboard to increase or decrease the IPD. They were instructed to find the value where it provided the most depth perception, yet visually comfortable to their eyes. For safety and making sure the visualization had minimum stereoscopic disparity, we restricted the IPD value to be between 0.1 and $8 \mathrm{~cm}$.

The experiment continued repeating the same task with the 3D model placed at 9 different levels of distance relative to the initial location of the airplane model $(95,75,50,25,0,-25,-50,-75$, and -95 units). The negative values mean the object is placed closer to the user, while the positive values mean it is further away relative to its initial location. While the participant repeated the task from the farthest distance to the nearest, the system recorded the IPD adjusted by the participant for each level of distance. The participants were allowed to take time as much as they needed for eye accommodation at each level of distance, and were allowed to take breaks if they felt eyestrain.

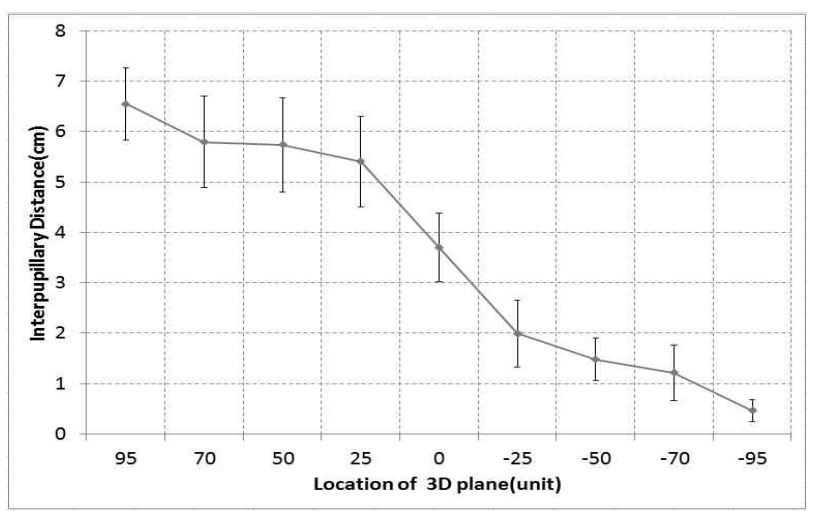

Figure 4. Average IPD chosen by the participants at each level of distance $(F=60.798, p<<0.001)$

\subsubsection{Results}

We had 12 participants whose ages were between 25 and 35 . The participants included 5 females and 7 males. Figure 4 shows the result of the first experiment with average value of the IPD chosen by the participants at each level of distance. As shown in the graph, the chosen IPD decreased as the 3D model got closer to the user. When the 3D model was located at -95 , which was the closest position in the experiment, the average IPD was approximately $0.4 \mathrm{~cm}$. Based on this result, we can confirm that as $3 \mathrm{D}$ scene gets closer to the user's viewpoint, the IPD has to be decreased to avoid visual discomfort.

\subsection{Experiment II}

\subsubsection{Experimental Procedure}

The second experiment was conducted to compare the proposed adaptive IPD adjustment method against using a fixed IPD and monoscopic visualization in terms of depth perception and visual discomfort while viewing an animated stereoscopic scene.

A short real-time animation of the airplane flying towards the user was built for the experiment. In the animation, the 3D airplane model moved from -110 to 110 units. The animation was presented to the participant in three different conditions: (1) monoscopic visualization, (2) stereoscopic visualization with fixed IPD $(6.5 \mathrm{~cm})$, and (3) stereoscopic visualization with adaptive IPD adjustment.

For applying the adaptive IPD adjustment method, we calculated the appropriate IPD corresponding to the location of the 3D object. We used the average value at each distance level calculated from the first experiment, and used linear interpolation for the positions in-between (or extrapolation for the points beyond the range). Based on this calculation the IPD was automatically set according to the position of the $3 \mathrm{D}$ object as the scene was animated.

The 3D airplane animation lasted for 10 seconds and it was played twice for each condition. After watching the animation under each condition, the participants were asked to answer a questionnaire.

\subsubsection{Results}

We recruited 6 participants ( 2 females and 4 males) for the second experiment, and all of them had previous experience with viewing 3D stereoscopic visualization.

For each condition, participants gave a rating using 5-point Likert scale (from 1: strongly disagree to 5 : strongly agree) to the four statements: 1) 'I felt like the airplane was moving towards me popping out of the screen.', 2) 'I perceived 3D depth of the scene.', 3) 'I thought that the scene looked natural.', and 4) 'I felt eyestrain.'

Figure 5 summarizes the results. Using Friedman tests with alpha level of 0.05 we found significant difference between the conditions for all four questions (felt airplane popping out: $\chi^{2}=10.3, p=0.006$, perceived 3D depth: $\chi^{2}=6.706, p=0.035$, looked natural: $\chi^{2}=7.000, p=0.03$, felt eyestrain: $\chi^{2}=8.667, p=0.013$ ). The proposed adaptive IPD adjustment method produced the highest rating in the first two questions, suggesting that adaptively reducing the IPD did not degrade the perceived depth illusion. On the other hand, the proposed method was perceived as natural as the monoscopic visualization, while reducing visual discomfort compared to using a fixed IPD. 


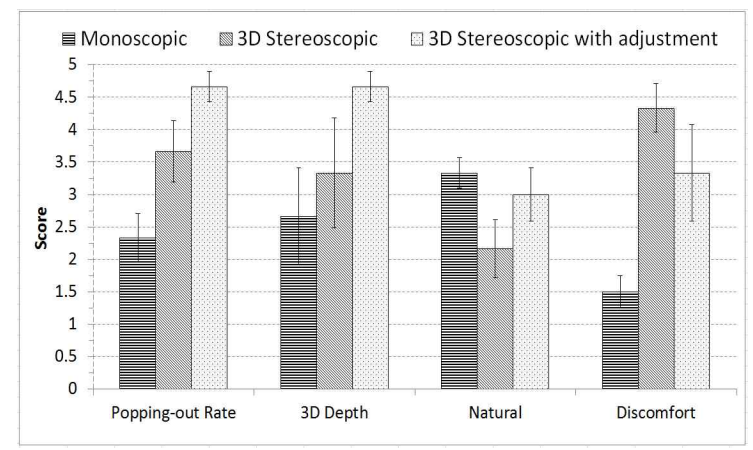

Figure 5. Results of the Likert scale rating

After experiencing all three conditions, the participants were asked to rank (1: least to 3 : most) the conditions based on their preference, and the amount of perceived depth illusion. Figure 6 shows that the adaptive IPD adjustment method was most preferred $\left(\chi^{2}=7.000, p=0.03\right)$ and it also showed the strongest perceived depth illusion $\left(\chi^{2}=7.000, p=0.03\right)$.

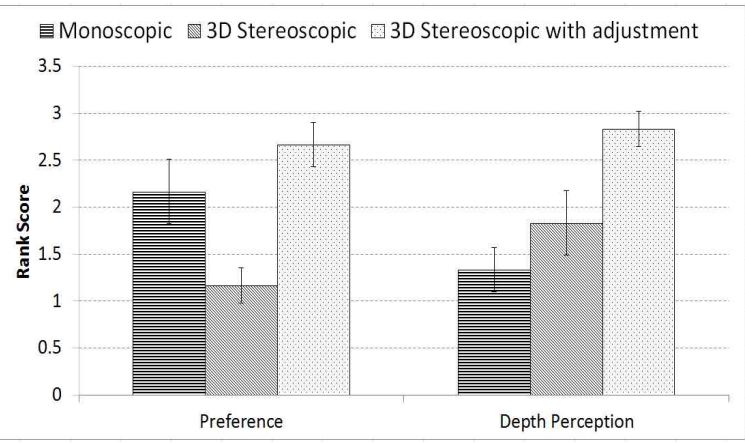

Figure 6. Average rank score of Preference and Depth Perception.

Note that the fixed IPD condition was the least preferred although it provided better depth perception over monoscopic visualization. This suggests that the visual discomfort caused by the fixed IPD might have degraded the overall user experience making it less preferred. In comparison, the proposed adaptive adjustment method reduced the visual discomfort while maintaining the depth illusion, hence making it a more preferable experience.

\section{CONCLUSION AND FUTURE WORK}

To reduce the visual discomfort in stereoscopic visualization caused by dynamically changed scene configuration, we proposed and investigated the adaptive IPD adjustment method which automatically adjusts the value based on the distance between the 3D scene and the user's viewpoint. Through two user experiments, we were able to confirm that the proposed method can reduce visual discomfort, yet maintain compelling depth perception, and as a result provide the most preferable $3 \mathrm{D}$ stereoscopic visualization experience.

For future work, we plan to improve the method to be applicable to more general stereoscopic visualization setup, and further investigate other factors that can be used as a metric for adaptively adjusting the IPD. Moreover, we will look into integrating a motion tracking system with the proposed method, which would provide a more immersive and realistic 3D stereoscopic viewing experience to the users. We will also have to run further experiments with a larger group of subjects to confirm the experimental results.

\section{REFERENCES}

[1] ITU-R. 2009. Features of three-dimensional television video systems for broadcasting. ITU-R Report BT. 2160, Geneva.

[2] Polonen, M., Jarvenpaa, T., and Bilcu. B. 2013. Stereoscopic 3D entertainment and its effect on viewing comfort: Comparison of children and adults. Applied Ergonomics 44, 1, 151-160.

[3] Johnson, A., Leigh, J., Morin, P., and Keken, P. V. 2006. GeoWall: Stereoscopic Visualization for Geoscience Research and Education. In Proc. of CGA '06, IEEE, 10-14.

[4] Pettersson, L. H., Spak, U., and Speipel, S. 2004. Collaborative $3 \mathrm{~d}$ visualizations of geo-spatial information for command and control. In Proc. of SIGRAD '04, 41-47.

[5] Häkkinen, J., Kawai, T., Takatalo, J., Leisti, T., Radun, J., Hirsaho, A., and Nyman, G. 2008. Measuring stereoscopic image quality experience with interpretation based quality methodology. In Proc. SPIE 6808, Image Quality and System Performance V, 68081B-1-68081B-12.

[6] Kaptein, R. G., Kuijsters, A., Lambooij, M. T. M., IJsselsteijn, W. A., and Heynderickx, I. 2008. In Proc. SPIE 6808, Image Quality and System Performance V, 680819-1680819-11.

[7] Tam, W. J., Speranza, F., Yano, S., Shimono, K., and Ono, H. 2011. Stereoscopic 3d-tv: Visual comfort. IEEE Transactions on Broadcasting 57, 2, 335-346.

[8] Mangait, S. and Gibson, J. 2012. Camera Placement for Handheld 3D Video Communications. In Conference Record of the $46^{\text {th }}$ Asilomar Conference on Signals, Systems and Computers (ASILOMAR) 2012, IEEE, 1731-1734.

[9] Ide, K., and Solpra, T. 2010. Adaptive Parallax for 3D Television. In 3DTV-Conference: The True Vision-Capture, Transmission and Display of $3 D$ Video (3DTV-CON), 1-4.

[10] Ide, S., Yamanoue, H., Okui, M., and Okano, F. 2002. Parallax distribution for ease of viewing in stereoscopic HDTV. In Proc. Stereoscopic Displays Virtual Reality Syst. IX, Vol. 4660, 38-45.

[11] Kishi, S., Kim, S. H., Shibata, T., Kawai, T., Häkkinen, J., Takatalo, J., Nyman, G. 2008. Scalable 3D image conversion and ergonomic evaluation. In Proc. SPIE 6803, Stereoscopic Displays and Applications XIX, 68030F-68030F-9.

[12] Konrad, J. 1999. Enhancement of viewer comfort in stereoscopic viewing: parallax adjustment. In Proc. SPIE 3639, Stereoscopic Displays and Virtual Reality Systems VI, 179-190.

[13] Park, J., Um, G., Ahn, C., and Ahn, C. 2004. Virtual control of optical axis of the 3DTV camera for reducing visual fatigue in stereoscopic 3DTV. ETRI Journal 26, 6, 597-604.

[14] Holliman, N. 2004. Mapping perceived depth to regions of interest in stereoscopic images. In Proc. SPIE 5291, Stereoscopic Displays and Virtual Reality Systems XI, 117128.

[15] Fehn, C. 2003. A 3D-TV approach using depth-image-based rendering (DIBR). In Proc. of Visualization, Imange, and Image Processing 2003. 